\title{
Rubber Band Recoil
}

\author{
By R. Vermorel, N. Vandenberghe and E. Villermaux $\dagger$ \\ Université de Provence, IRPHE, 49, rue Frédéric Joliot-Curie \\ 13384 Marseille Cedex 13, France
}

When an initially stretched rubber band is suddenly released at one end, an axialstress front propagating at the celerity of sound separates a free and a stretched domain of the elastic material. As soon as it reaches the clamped end, the front rebounds and a compression front propagates backward. When the length of the compressed area exceeds Euler critical length, a dynamic buckling instability develops. The rebound is analysed using Saint-Venant's theory of impacts and we use a dynamical extension of the Euler-Bernoulli beam equation to obtain a relation between the buckled wavelength, the initial stretching and the rubber band thickness. The influence of an external fluid medium is also considered: due to added mass and viscosity the instability growth rate decreases. With a high viscosity, the axialstress front spreads because of viscous frictional forces during the release phase. As a result, the selected wavelength increases significantly.

Keywords: Rubber band, elastic instability, dynamic buckling

\section{Introduction}

In his classical treatment of the elastica, Euler (1744) proved that for a given length and for given boundary conditions, there exists a critical load at which a rod buckles. Among the different possible bent shapes, only the one with the smallest number of inflections is stable i.e. the shape corresponding to the fundamental flexural mode (see e.g. Love (1944)). Thus the only characteristic length associated with the buckling instability is the length of the rod itself.

However, when a compressive load several times higher than the Euler critical force is suddenly applied to an elastica at rest, the buckling instability develops dynamically, and a characteristic wavelength is selected. Lindberg (1965) proposed a simple model to predict the amplified wavelength. Using Euler-Bernoulli theory for the elastic beam, he studied the growth of the different flexural modes of the beam. The theory predicts that the most amplified wavelength decreases like the inverse square root of the compression strain. Lindberg also devised an ingenious way to determine the buckled wavelength from experiments on metallic elastic beam and on rubber bands. In the case of the metallic beam he found a fair agreement with theory, while in the case of the rubber band, discrepancies were stronger: the measured wavelength was 70 percent higher than predicted for reasons that were not elucidated.

The aim of the present work is to study in details the dynamic buckling instability responsible for the recoil of a rubber band. Indeed the rubber band is an interesting system for the study of dynamic buckling because the characteristic

$\dagger$ Also at: Institut Universitaire de France 
speed of sound in rubber is moderate (about $40 \mathrm{~m} \mathrm{~s}^{-1}$ ) and strains and displacements can be large. It also represents a simple case study of more general situations where flexural waves and compression waves are coupled, as those encountered in the related problem of brittle rods fragmentation under impact (Gladden et al. (2005)). The question is envisaged in its most general setting, including the influence of a surrounding medium as we perform experiments in air, and in liquids, namely water and water-glycerol mixtures to investigate the effect of added mass and fluid viscosity.

\section{Experimental set-up}

We first consider the recoil of a cantilever rubber band. One end of the rubber band is firmly clamped on the experimentation table. The operator holds the free end, stretches the elastic to the desired length, and releases it suddenly. A setup was also designed that allowed to stretch and release the rubber band from both ends simultaneously to study the influence of the boundary conditions. A thin fishing line is glued on both ends of the elastic in such a way that the line and the elastic material form a loop. This loop is placed around two pulley. Thus the operator can stretch and release the rubber band just by pulling and dropping the fishing line.

The elastics were cut from natural latex rubber sheets of different thicknesses from $0.254 \mathrm{~mm}$ up to $1.270 \mathrm{~mm}$. The characteristic length and width of the rubber bands are $l_{0}=150 \mathrm{~mm}$ and $b=4 \mathrm{~mm}$. Measuring the force-extension curve, the static stretching test reveals that in the range of stretching between 0 and 100 percent, the elastic behavior of the rubber remains linear (within 3 percent) with a Young modulus $E=1.5 \mathrm{MPa}$ and no significant hysteretic behaviour i.e. or stress softening of the rubber (Bouasse and Carrière (1903), also called Mullins effect after Mullins (1947)) were observed. For higher stretching, significant deviation from the ideal Hookean behavior was observed and in most experiments the stretching has been limited to the range 0 to 100 percent. The density of the rubber is $\rho=$ $990 \mathrm{~kg} \mathrm{~m}^{-3}$ and thus the nominal wave speed for longitudinal disturbances is $c=$ $(E / \rho)^{1 / 2}=39 \mathrm{~m} \mathrm{~s}^{-1}$.

All diagnostics are based on quantitative image analysis, resolved in time, using a Phantom V5 high-speed video camera to record movies at typical frame rates of 10,000 up to 30,000 frames per second. The rubber band is illuminated from behind using a white light source and a diffusing screen or by direct lighting using a black or white background. Regularly spaced marks are drawn on the elastic to follow the motion of the material points.

In order to study the influence of the external medium, experiments were also conducted with the set-up immersed in a tank filled with water or with waterglycerol mixtures of controlled viscosity. Viscosities of the mixture were measured using a Couette viscosimeter and we used viscosities from $\eta=1.010^{-3} \mathrm{~Pa}$ s (pure water) up to $\eta=6.510^{-1} \mathrm{Pas}$. 

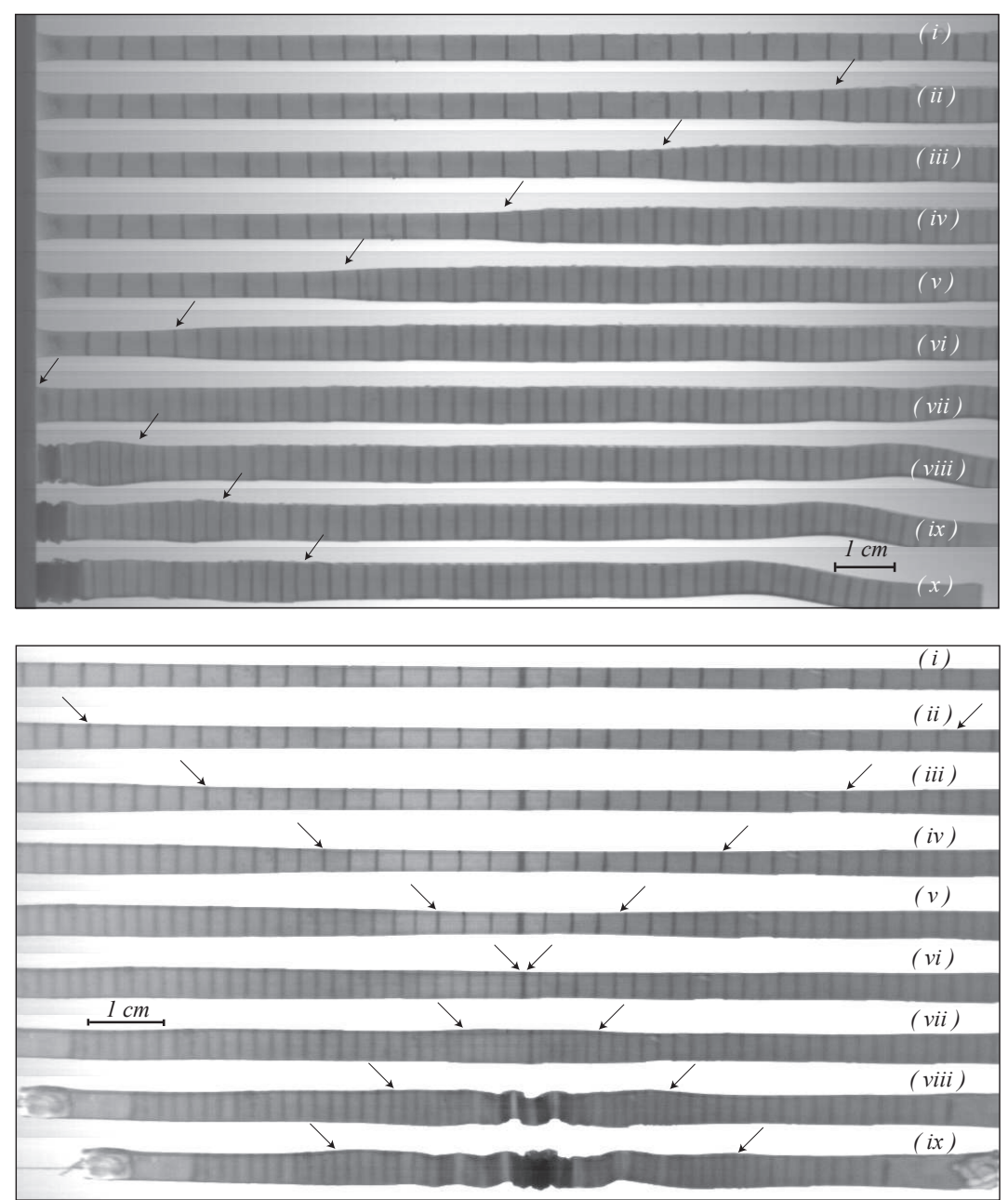

Figure 1. Top: front propagation in a clamped rubberband with initial stretch $\epsilon_{0}=1$. Arrows mark the front position. ( $i)$ to $(v i)$ The front propagates towards the clamped end and drags the free region. (vii) When the front reaches the clamped end, the strain-free rubber band moves towards the clamped end. (viii) to $(x)$ After impact, a compressive front propagates backward and triggers a dynamic buckling instability. Time goes from top to bottom by steps of $350 \mu \mathrm{s}$. A movie showing the front propagation is included in the electronic supplementary materials. Bottom: fronts propagation in a rubber band simultaneously released from both ends. The front propagate towards the middle of the elastic. The initial stretch is $\epsilon_{0}=1$. Arrows mark the positions of the fronts. $(v i)$ when the fronts cross each other, compressive fronts set out from the middle $((v i i)$ to $(i x))$ and trigger buckling. Time goes from top to bottom by steps of $320 \mu \mathrm{s}$.

\section{Recoil of a rubber band in air}

\section{(a) Phenomenology}

Stretching and releasing a rubber band is a common experience. The typical timescale of this phenomenon is $l_{0} / c \approx 3.8 \mathrm{~ms}$, hence the use of high speed imaging. 

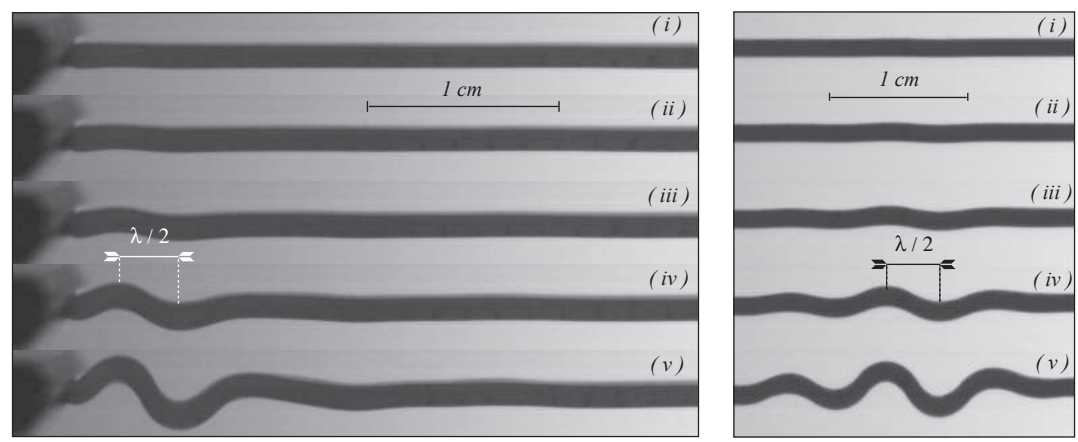

Figure 2. Left: Early stages of the dynamic buckling of a clamped rubber band. Time goes from top to bottom by steps of $117 \mu \mathrm{s}$. The initial stretch is $\epsilon_{0}=0.3$. Right: Early stages of the dynamic buckling of a rubber band simultaneously released from both ends. Time goes from top to bottom by steps of $130 \mu \mathrm{s}$. The initial stretch is $\epsilon_{0}=0.2$. A movie showing the dynamic buckling is included in the electronic supplementary materials.

When the tension is suddenly released, a front propagates towards the clamped end (see figure 1, images $(i)$ to $(v i)$ ) at the celerity $c$ in the material. The front separates two regions: a stress-free area between the free end and the front, and a stretched area between the front and the clamped end. As the front propagates it drags the free region down to the clamped end at velocity $V$, which is a fraction of $c$. When the front reaches the clamped end, the whole rubber band is free, moving towards the table at velocity $V$ (figure 1 (vii)).

The configuration is then equivalent to a free rubber band moving at velocity $V$ impacting a rigid surface (see Saint-Venant and Flamand (1883) and references therein). A compressive front propagates backward (i.e. towards the free end) at speed $c$ in the frame of the rubber band (figure 1 (viii) to $(x)$ ). Between the clamped end and the front, the elastic is compressed. As soon as the compressive front has covered a critical distance from the clamped end, the compressive stress is applied to a region long enough to trigger off a buckling instability. The elastic starts to bend with a well defined wavelength (figure $2(i v)$ ). The first complete half wavelength appearing during the dynamic buckling instability will be refered to as the half buckled wavelength. In later times the front propagates towards the free end inducing more bending of the rubber band. We will focus on the first half wavelength only, because the subsequent dynamics becomes more complicated. In particular, near the clamped end, the transverse displacement resulting from the buckling is coupled to the propagation of the longitudinal wave.

To check the influence of the boundary conditions on the dynamics, we performed a similar experiment with a rubber band simultaneously released from both ends. Two fronts propagate towards the middle of the elastic. When the fronts reach the middle, the rubber band is stress-free but its two halves are moving with opposite velocities $V$ and $-V$. The configuration is then equivalent to the classical problem of two rods impacting each other. Compressive fronts propagate away from the junction (i.e the middle of the rubber band), triggering the dynamic buckling instability in both sides of the rubber band and a selected wavelength is observed. Measurements show that the dynamics is strictly identical to the case of 
( $i$ )

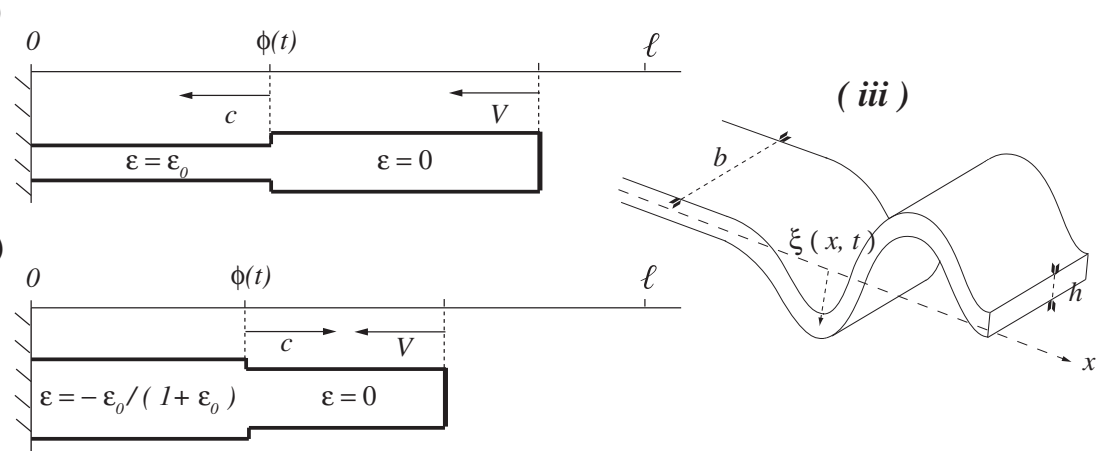

Figure 3. (i) Schematic of the stress free front propagation. $\ell$ is the length of the stretched rubber band. $\phi$ is the position of the front. The stress-free front propagates towards the clamped end at speed of sound $c$ and it drags the free region with a constant velocity $V$. (ii) The rebounding front propagating backward. (iii) Schematics of the dynamic buckling of a rubber band. $b$ and $h$ are the width and the thickness of the band. $\xi$ is the transverse displacement, function of the longitudinal coordinate $x$ and on the time $t$.

the clamped rubber band for low stretching (less than $50 \%$ ). However, for higher stretching, the friction of the fishing line as it slides against the axes results in a slight decrease of the velocity of the free regions of the rubber band. Therefore all the measurements reported in this paper were obtained with the more reliable clamped-free configuration.

\section{(b) Compression front}

The rubber band is modeled as a hookean elastic rod experiencing small strain. We neglect the effect of lateral inertia in the propagation and we use the small strain hypothesis. Thus longitudinal perturbations are governed by the linear wave equation with the propagation speed $c$.

Let $\ell$ be the length of the stretched rubber band and $\ell_{0}$ its length at rest. The initial strain $\epsilon_{0}$ is

$$
\epsilon_{0}=\frac{\ell-\ell_{0}}{\ell_{0}}
$$

The front is a discontinuity that separates a strain-free region and a stretched region in which $\epsilon=\epsilon_{0}$ (figure 3 (i)). The front propagates at speed $c$ and it reaches the anchor point at time $t_{i}=\ell / c$. Then the elastic is strain-free and its length is $l_{0} . V$ being the speed of the free end, we have $\ell-\ell_{0}=-V t_{i}$ and thus we obtain that

$$
V=-\left\{\frac{\ell-\ell_{0}}{\ell}\right\} c=-\left\{\frac{\epsilon_{0}}{1+\epsilon_{0}}\right\} c
$$

This relation holds for all material points in the free region.

When the free front reaches the clamped end, the whole rubber band is strainfree and translates at speed $V$. Thus the problem is equivalent to a rubber band impacting a rigid surface at speed $V$. Let $\zeta(x, t)$ be the longitudinal displacement in the rubber band. $x$ is the coordinate of a material point along the rod with $x=0$ being the anchor point. When the front reaches the $x=0$ position, all the 

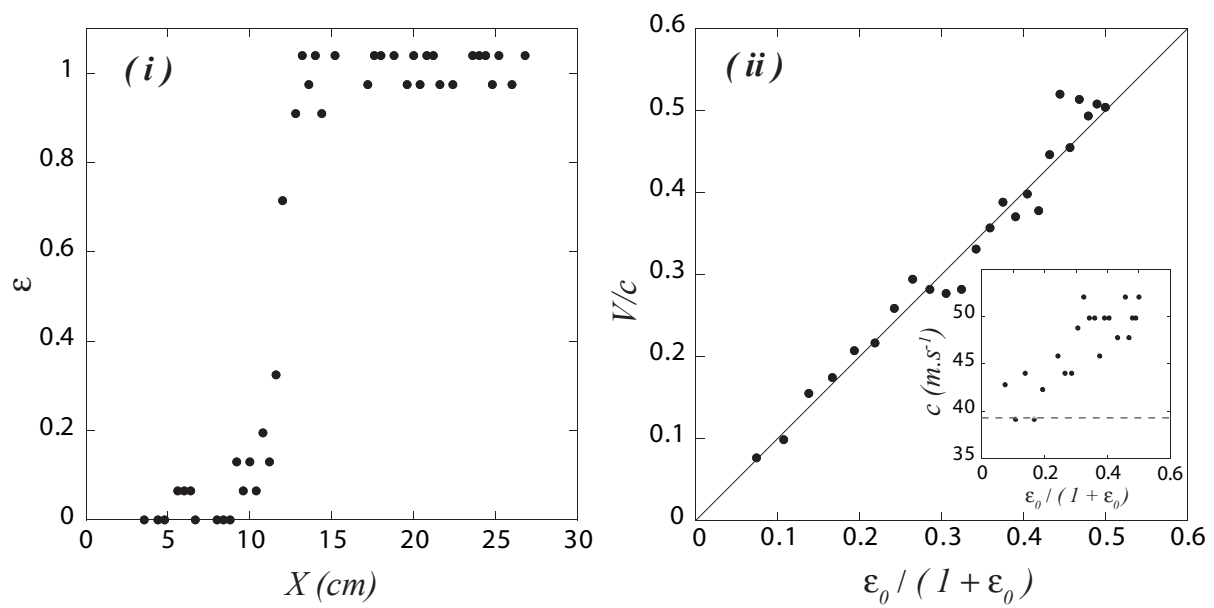

Figure 4. (i) Stretch in the rubber band during the propagation of the stress free front. $x$ is the material coordinate. (ii) Ratio $V / c$ versus $\epsilon_{0} /\left(1+\epsilon_{0}\right)$. The dots stand for the measurements, the line stands for the theoretical prediction with no adjusted parameters. Note that here $c$ is the measured front speed which is slighthly higher than $(E / \rho)^{1 / 2} \approx 39 \mathrm{~m} \mathrm{~s}^{-1}$ (see insert).

points of the elastic are moving at speed $V$. At time $t=0$, the front suddenly encounters a $\zeta(0, t)=0$ condition. It rebounds and propagates backward at speed $c$ (in the material frame i.e. it is propagating at speed $c+V$ in the laboratory frame with $V<0$ ). The front propagating away from the "impact point" separates a compressed area in which the speed is zero and a stress free area moving at speed $V$ (figure $3(i i))$. At time $t$, the front has reached the point $x=\phi=c t$ and this point has been displaced by a quantity $\zeta(x, t)=V t=V x / c$. Thus the strain in the compressed area is

$$
\epsilon=V / c=-\epsilon_{0} /\left(1+\epsilon_{0}\right) .
$$

Behind the rebounding front, the compressive force is given by Hooke's law

$$
T=\sigma A=-E A \epsilon_{0} /\left(1+\epsilon_{0}\right)
$$

where $A=b h$ is the cross section area if $b$ and $h$ are the width and thickness of the rubber band.

\section{(c) Mode selection}

We introduce an equation for the transverse displacement $\xi(x, t)$ (figure $3(i i i)$ ) by plugging the above compressive force into the equation describing the dynamics of the bending waves (see also Gladden et al. (2005)) and using Euler-Bernoulli hypotheses.

$$
\rho b h \frac{\partial^{2} \xi}{\partial t^{2}}-\frac{\partial}{\partial x}\left(T \frac{\partial \xi}{\partial x}\right)+E I \frac{\partial^{4} \xi}{\partial x^{4}}=0
$$

where $I=b h^{3} / 12$ is the flexural inertia momentum in the flexion plane. 
We look for solutions of the form $\xi(x, t)=\xi_{0} \exp (i k x-i \omega t)$. With $T$ constant along the rod, the dispersion relation reads

$$
\omega^{2}=\frac{E I}{\rho b h} k^{2}\left\{k^{2}+\frac{T}{E I}\right\}
$$

For a compressive force, $T$ is negative. Unstable modes have wave numbers in the range 0 to $k_{c}$ where $k_{c}$ is the marginal wavenumber,

$$
k_{c}=\sqrt{\frac{|T|}{E I}}
$$

The most amplified wave number is $k_{m}=k_{c} / \sqrt{2}$, so that, making use of equation (3.4) for $T$, the most amplified wavelength writes, Mutatis Mutandis

$$
\lambda_{m}=\pi h \sqrt{\frac{2}{3}} \sqrt{\frac{1+\epsilon_{0}}{\epsilon_{0}}}
$$

and its associated growth rate is

$$
\sigma_{m}=\sqrt{3} \frac{\epsilon_{0}}{1+\epsilon_{0}} \frac{c}{h}
$$

The selected mode depends on both the material elastic properties and intensity of the compression, but since the compression is itself a function of the material elasticity, a cancellation effect makes $\lambda_{m}$ depend on geometrical parameters only, namely the thickness of the rubber band and initial stretching.

Of course, this naive expectation assumes that the compression front has travelled by a distance at least equal to $\lambda_{m}$ during a time lapse given by $\sigma_{m}^{-1}$. A more general mode selection criterion would thus be that the amplified wavenumber $k$ is the one for which

$$
\tau(k) c \simeq k^{-1}
$$

and $k=k_{m}$ otherwise if $\tau(k) c \gg k^{-1}$. There, $\tau(k)$ is the instability timescale associated with $k$ through the dispersion relation (3.6) such that $\tau(k)^{-1}=\operatorname{Re}\{-i \omega\}$.

The above reasonings are made within the long wave approximation $(k h \ll 1)$ and disregard three dimensional effects when the wavelength becomes of the order of the thickness $h$, as it is nevertheless the case for the higher initial elongations $\epsilon_{0}$. We also do not account for any coupling between the instability development and the compression force, nor any nonlinear elastic response of the material.

\section{(d) Experimental results}

To measure the propagation speeds, we draw regularly spaced marks along the rubber band (figure 1). The theoretical value of the front velocity for the rubber bands that we used in the experiments is $39 \mathrm{~m} \mathrm{~s}^{-1}$, which is in agreement with the measurements for small initial stretching. However, the speed of the front is slightly higher, typically $50 \mathrm{~m} \mathrm{~s}^{-1}$, in particular for high initial stretching $\left(\epsilon_{0} \geq 0.6\right)$. This is likely due to the effect of the strain rate on the elongational modulus known in rubber (Kolsky 1949), not taken into account here. 

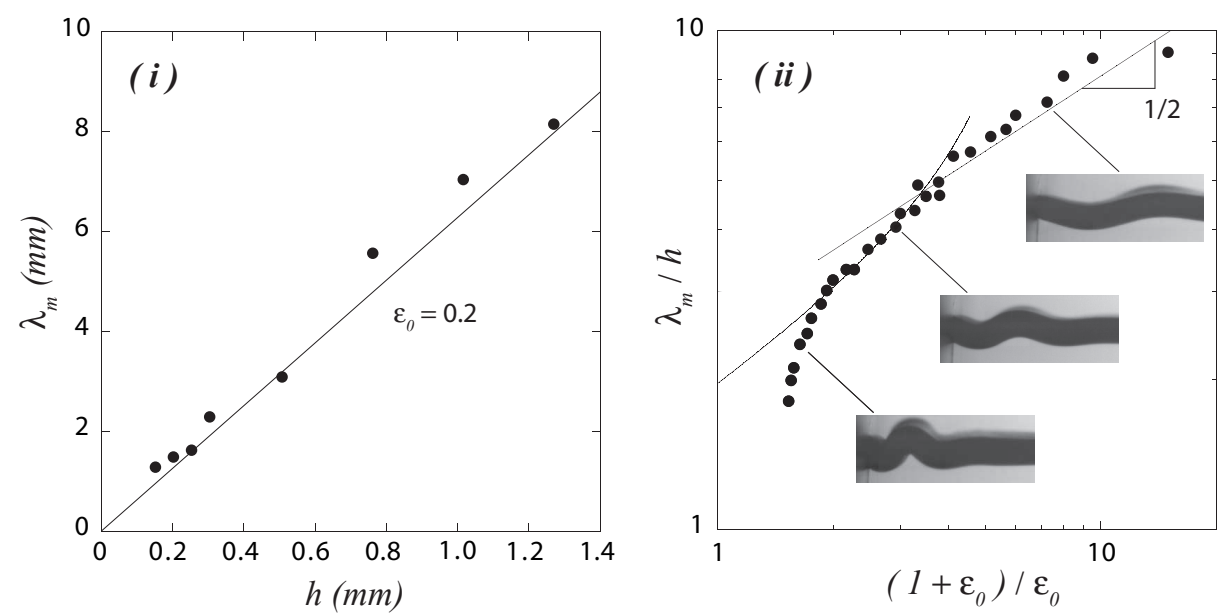

Figure 5. (i) Variation of the selected wavelength $\lambda_{m}$ with the thickness of the rubber band $h$. The straight line stands for the theoretical curve with no adjusted parameters. (ii) Variation of the selected wavelength $\lambda_{m}$ with the initial stretch $\epsilon_{0}$. The straight line stands for the theoretical curve from equation (3.8) with no adjusted factor. The curve fit is from equation (3.10) with $\tau(k) c=2.6 k^{-1}$.

The measured value of $V$ typically ranges from $4 \mathrm{~m} \mathrm{~s}^{-1}$ to $20 \mathrm{~m} \mathrm{~s}^{-1}$, depending on the initial stretch (figure $4(i i)$ ). The evolution of the ratio $V / c$ ( $c$ is the measured front speed) is in fair agreement with the theoretical prediction. Measurements of the stretching profile show that the front shape is well approximated by a step function (figure $4(i)$ ). Actually, the dependency of $V$ on initial stretch $\epsilon_{0}$ found in section (b) is valid even for high initial stretching $\left(\epsilon_{0} \simeq 1\right)$, i.e. beyond the limitations of the small strain hypothesis.

The first selected wavelength was obtained for rubber bands of thickness from $0.254 \mathrm{~mm}$ up to $1.27 \mathrm{~mm}$, for an initial stretch $\epsilon_{0}=0.2$ (figure $5(i)$ ). All properties and dimensions of the rubber bands are the same but their thickness. We find that $\lambda_{m} \sim h$ as expected. Figure 5 (ii) shows the experimental wavelengths obtained with a rubber band of thickness $h=1.27 \mathrm{~mm}$. Experimental results agree with the prediction from equation (3.8) for small initial stretching (i.e for high $\left(1+\epsilon_{0}\right) / \epsilon_{0}$ ratio). However for large initial stretch (i.e. for $\left(1+\epsilon_{0}\right) / \epsilon_{0}$ smaller than 4$)$ the measured wavelengths are shorter than predicted from (3.8). A better fit is obtained considering a mode selection criterion based on the length travelled by the recompression front given in equation (3.10). For even higher initial stretch, (i.e. for $\left.\left(1+\epsilon_{0}\right) / \epsilon_{0}<2\right)$, the wavelength becomes of the order of the band thickness and the long wave approximation breaks down.

Note that using a dispersion relation that includes both Rayleigh's correction (rotational inertia) and Timoshenko's correction (effect of shear stress) leads to even higher wavelengths (see e.g. Graff, (1975)). Finaly, once the band is wrinkled, the axial stress relaxes by a simple geometrical effect, as suggested by figure 1 (viii) and $(i x)$ ). This leads to a coarsening of the initial wrinkled pattern, a phenomenon which is probably at the origin of Lindberg's strong discrepancy between the anticipated and measured wavelength. 


\section{Recoil of a rubber band in fluids}

Several new effects are expected in the presence of an external fluid. First when the instability develops, fluid must be moved together with the rod and thus we expect added mass effects. Moreover, if the fluid is viscous, we expect damping. In this section we modify the analysis of section 3 to account for these ingredients. As we shall see, in order to accurately describe the dynamic of the rubber band in a fluid, we must also consider the effect of viscosity on the axial stress front propagation.

\section{(a) Modification of the instability}

We consider a thin rod under compressive stress surrounded by an external fluid. We use the same hypotheses and notations as in section 3 (b). The fluid is newtonian and incompressible, of density $\rho_{f}$ and of kinematic viscosity $\nu$. The dispersion equation in non-dimensional form (see Appendix A) reads

$$
\left(k_{*}+4 M\right) \sigma_{*}^{2}+4 \chi k_{*}\left[k_{*}+\left(\frac{M}{\chi} \sigma_{*}+k_{*}^{2}\right)^{1 / 2}\right] \sigma_{*}+4 k_{*}^{3}\left(k_{*}^{2}-1\right)=0
$$

where $k_{*}=k / k_{c}$ and $\sigma_{*}=\sigma / \sigma_{m} . M$ and $\chi$ are two non-dimensional numbers related to the added mass term and the viscous term, defined as

$$
M=\frac{\rho_{f}}{4 \pi \rho k_{c} h}, \quad \chi=\left[\frac{\eta^{2} b}{\rho|T| h}\right]^{1 / 2}
$$

where $T=-E b h \epsilon . \chi$ depends on the dynamic viscosity $\eta=\rho_{f} \nu$ of the fluid and on the Young modulus $E$, density $\rho$, thickness $h$ and extensional strain of the rod (but $b$ cancels out in the expression for $\chi$ when $T$ is expressed in terms of $E$ ).

Figure 6 shows the dispersion relation obtained in different fluids (note that the glycerol we used was not pure and its dynamic viscosity was $\eta=0.65 \mathrm{~Pa}$ s). The viscous number $\chi$ is rather small ( $\chi=0.02$ for a dynamic viscosity $\eta=0.65 \mathrm{~Pa} \mathrm{~s}$ ). Therefore, the main effect of the external fluid is the added mass effect that results in a significant decrease of the instability growth rate. Compared to the theoretical value in vacuum, growth rate should decrease by a factor 1.6 for water and almost a factor 2 for glycerol. On the other hand, the selected wavelength is not significantly modified by the interaction with the external fluid. For high $\chi$ numbers (i.e for high viscosity), the selected wave number decrease to an asymptotic value, $k_{* m} \sim 1 / \sqrt{3}$ instead of $k_{* m}=1 / \sqrt{2}$ with no external medium. The added mass tends to increase the selected wavenumber anf for high values of M, $k_{* m}$ goes to $\sqrt{3 / 5}$.

\section{(b) Experiments in fluids}

We conducted experiments in water, and in water-glycerol mixtures with viscosity ranging from $\eta=410^{-3} \mathrm{~Pa}$ s up to $\eta=0.65 \mathrm{~Pa}$ s. Figure 6 (ii) shows wavelengths measured in air, water and water-glycerol mixtures. In water the results are similar to those performed in air. With more viscous fluids we observe a significant increase of the selected wavelength. The wavelength is more than doubled in the glycerol $(\eta=0.65 \mathrm{~Pa} \mathrm{~s})$. As discussed above, that effect is too large to be attributed to the impact of viscosity on mode selection in the buckling instability. 

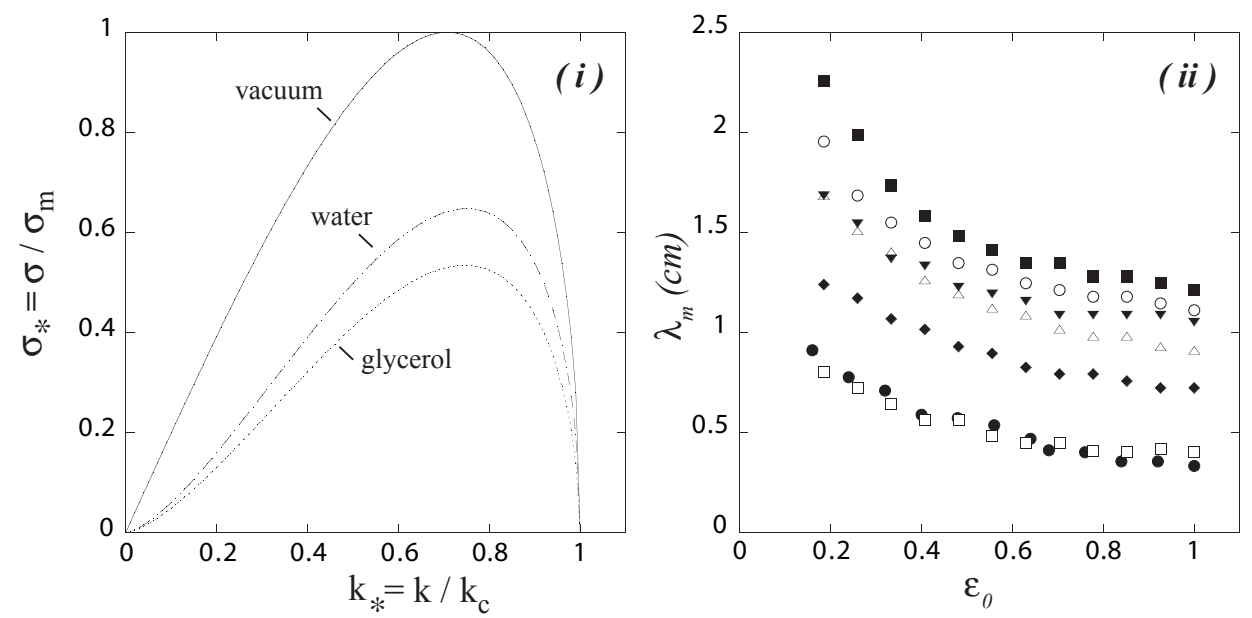

Figure 6. $(i)$ Predicted non-dimensional growth rate $\sigma_{*}$ versus nondimensional wave number $k_{*}$. For vacuum $\chi=0$ and $M=0$, for water $\chi=3.510^{-5}$ and $M=0.25$, for glycerol $\chi=210^{-2}$ and $M=0.35$. (ii) Measurements of the selected wavelength $\lambda_{m}$ vs initial stretch $\epsilon_{0}$ for different fluids: •, air ; $\square$, water ; $\diamond$, glycerol $\eta=0.04 \mathrm{~Pa} \mathrm{~s} ; \Delta$, glycerol $\eta=0.190 \mathrm{Pas} ; \mathbf{\nabla}$, glycerol $\eta=0.310 \mathrm{~Pa} \mathrm{~s} ; \circ$, glycerol $\eta=0.450 \mathrm{~Pa} \mathrm{~s} ; \mathbf{\square}$, glycerol $\eta=0.650 \mathrm{Pas}$.

To clarify the effect of viscosity on the propagation of the compression front, we visualized the flow field. Particles were added to the fluid (glycerol with dynamic viscosity $\eta=0.65 \mathrm{~Pa} \mathrm{~s}$ ) and the elastic was illuminated by a laser sheet. After the release of the rubber band, we observed the development of a boundary layer following the axial-stress front. The spatial profile of the boundary layer in the plane of the length and thickness of the rubber band (which was the plane illuminated by the laser sheet) is shown on figure $7(i)$. The profile of the boundary layer is well fitted by a square root function of the axial coordinate. A movie showing the development of the boundary layer is included in the electronic supplementary materials.

Let $U(x, y, t)$ be the velocity profile in the fluid at a given instant of time and axial location. Direction $y$ is perpendicular to the band surface located in $y=0$. Figure $7(i i)$ shows of such a profile. The viscous frictional force per unit length of the band is given by

$$
\tau_{f}=-2 b \eta\left(\frac{\partial U}{\partial y}\right)_{y=0}
$$

the factor 2 accounting for the two sides of the band. Using the velocity gradient measured on figure $7(i i)$ and $\ell_{0}$ as an estimate of the length the front has gone through, an order of magnitude of the frictional force is $F_{f} \sim \tau_{f} \ell_{0} \simeq 0.5 \mathrm{~N}$. A typical value of the velocity of the free end of the rubber band is $6 \mathrm{~m} \mathrm{~s}^{-1}$. Thus, the order of magnitude of the drag force $F_{d} \simeq b \eta U$ at the free end of the rubber band is $F_{d} \simeq 1.510^{-2} N$. Obviously, the drag force at the end is small compared to the frictional force. Moreover the friction increases as the stress free front propagates and as the region dragged by the front gets wider. 

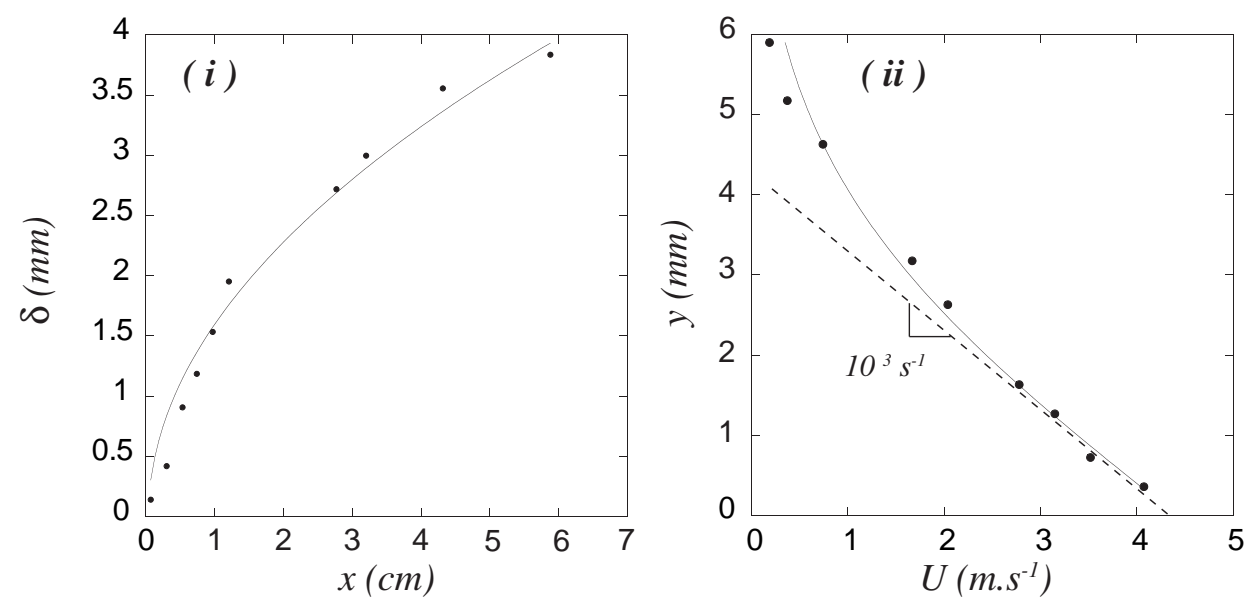

Figure 7. (i) Thickness of the boundary layer $\delta$ versus axial coordinate $x$ at one instant of time. The origin for $x$ is the position of the stress free front. The continuous line is a square root fit. (ii) Velocity profile in the boundary layer at the position $x=85 \mathrm{~mm} 13 \mathrm{~ms}$ after the passage of the front. Data is fitted by a function of the form $U_{0}(1-\operatorname{erf}(y / \delta))$ and we find $\delta=4.74 \mathrm{~mm}$, which is close to the theoretical prediction $(4 \nu[t-x / c])^{1 / 2}=4.89 \mathrm{~mm}$.

An equation for the longitudinal displacement $\zeta$ accounting for frictional forces is

$$
\rho b h \frac{\partial^{2} \zeta}{\partial t^{2}}=E b h \frac{\partial^{2} \zeta}{\partial x^{2}}+\tau_{f}
$$

In this plane boundary layer approximation, we first neglect the contribution of the small dimension $h$ and disregard the contribution of the corners. Let $U_{0}(x, t)=$ $U(x, 0, t)=\partial \zeta(x, t) / \partial t$ be the axial velocity of the band. When $U_{0}$ is a function of time, the net force par unit length applied to the band is (see Stokes, 1850 cited in Lamb, 1932)

$$
\tau_{f}=-\frac{2 \eta b}{\sqrt{\pi \nu}} \int_{0}^{\infty} \frac{\partial U_{0}}{\partial t}\left(t-t^{\prime}\right) \frac{\mathrm{d} t^{\prime}}{\sqrt{t^{\prime}}}
$$

so that equation 4.4 becomes

$$
\rho h \frac{\partial^{2} \zeta}{\partial t^{2}}=E h \frac{\partial^{2} \zeta}{\partial x^{2}}-\frac{2 \eta}{\sqrt{\pi \nu}} \int_{0}^{\infty} \frac{\partial^{2} \zeta}{\partial t^{2}}\left(t-x / c-t^{\prime}\right) \frac{\mathrm{d} t^{\prime}}{\sqrt{t^{\prime}}}
$$

where $t$ has been replaced by $t-x / c$ (where $c$ is the velocity of the front) because the viscous term vanishes for $t<x / c$, i.e when the front has not reached the material point $x$ yet.

Now, this integro-differential equation can be simplified by considering figure 7 suggesting that the transverse velocity profile in the fluid is, in fact, very close to that developing over a plate initially at rest and moved suddenly at $t=x / c$ at a constant velocity (Stokes, 1850)

$$
U(x, y, t) \approx U_{0}(x, t)\left\{1-\operatorname{erf}\left(\frac{y}{\sqrt{4 \nu(t-x / c)}}\right)\right\}
$$



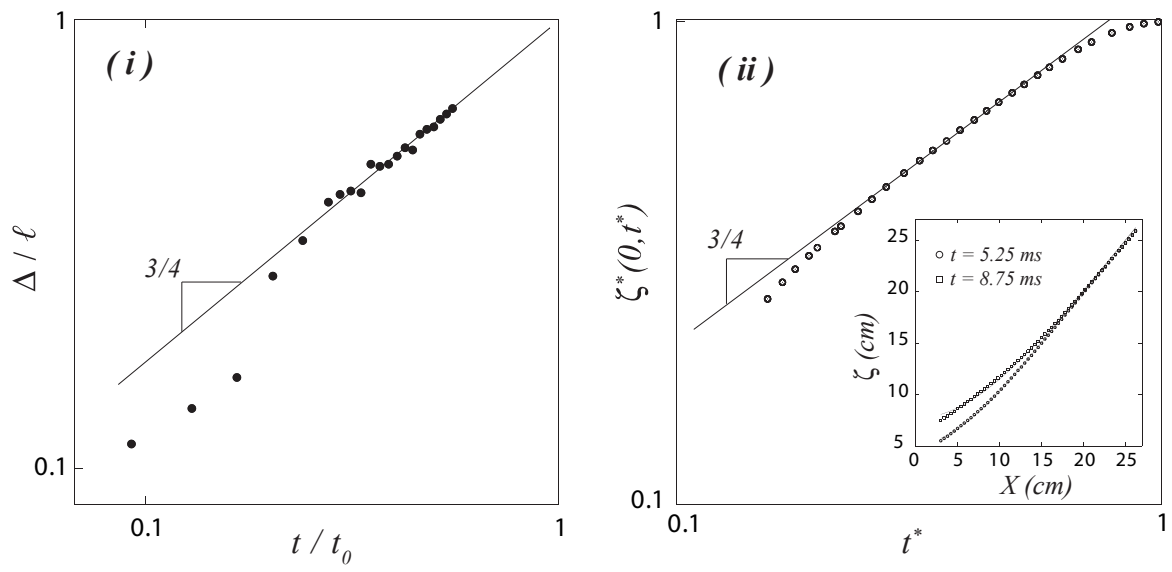

Figure 8. (i) Dimensionless width $\Delta / l$ versus dimensionless time $t / t_{0} . l$ is the length of the stretched rubber band, $t_{0}$ is the time such that $D t_{0}^{3 / 4}=l^{2} . \Delta$ is obtained by fitting the strain front profile (see text) (ii) Dimensionless displacement at the free end vs dimensionless time. The front profile is well fitted by expression (4.11) (insert) and displacement of the free end goes like $t^{3 / 4}$ at large times.

Then, using equation (4.7), we obtain in place of (4.4)

$$
\rho h \frac{\partial^{2} \zeta}{\partial t^{2}}=E h \frac{\partial^{2} \zeta}{\partial x^{2}}-\frac{2 \eta}{\sqrt{\pi \nu(t-x / c)}} \frac{\partial \zeta}{\partial t}
$$

This equation has no analytic solution. However, if we focus on the long time dynamics for $t \gg x / c$ (far from the front and close to the released end) and therefore neglect inertia, an asymptotic solution can be found. Equation (4.8) being linear, the stretch $\epsilon=\partial \zeta / \partial x$ obeys the same equation as the longitudinal displacement $\zeta$, thus, in the above mentioned limit

$$
\frac{\partial \epsilon}{\partial T}=D \frac{\partial^{2} \epsilon}{\partial x^{2}}
$$

where $D=E h \sqrt{\pi \nu} /(3 \eta)$. We use the time scale $T=t^{3 / 2}$ and consider that $\epsilon(0, t)=$ 0 . Then the asymptotic solution is

$$
\frac{\epsilon(x, T)}{\epsilon_{0}}=\operatorname{erf}\left\{\frac{x}{2 \sqrt{D T}}\right\}
$$

which implies that the width of the front scales like $T^{1 / 2}=t^{3 / 4}$ for large propagation time. Integrating $\epsilon(x, T)$ with respect to $x$, we find the expression for the displacement $\zeta$ :

$$
\zeta(x, T)=\epsilon_{0} x \operatorname{erf}\left\{\frac{x}{2 \sqrt{D T}}\right\}+\frac{2 \epsilon_{0} \sqrt{D T}}{\sqrt{\pi}} \exp \left\{-\frac{x^{2}}{4 D T}\right\}
$$

which implies that the displacement of the free end of the rubber band goes like $T^{1 / 2}=t^{3 / 4}$ for large propagation time. The consistency of the inertialess approximation is justified a posteriori noticing that the terms retained in the balance of 

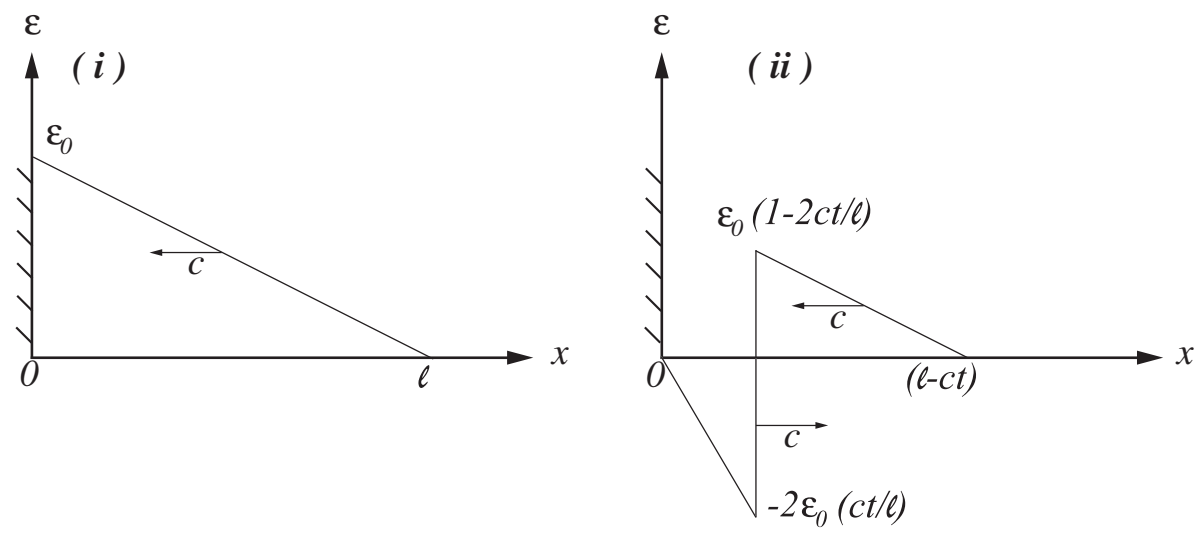

Figure 9. (i) Linear strain profile reaching the clamped end with velocity c. (ii) Strain profile at time $t$ after the rebound at the clamped end.

equation (4.9) are both of order $\epsilon T^{-1}$ while the inertial term is of order $\epsilon T^{-4 / 3}$, i.e. subdominant at large time.

The displacement and the strain were measured by tracking the motion of marks drawn on the rubber band. For different values of time, the strain profile is fitted by a function $\operatorname{erf}(x / \Delta)$ and the values of $\Delta$ are plotted on figure 8(i). For sufficiently large time we observe the expected behavior $\Delta \sim t^{3 / 4}$. The displacement front is well fitted by expression (4.11) and that of the free end goes like $t^{3 / 4}$ at large times.

In all cases however, the apparent coefficient in front of $\sqrt{D t}$ was about half the expected one. This deviation indicates that the experimental friction is larger than the one anticipated by approximating the total friction as the sum of the two boundary layers friction on both sides of the band (equations (4.3) and (4.4)). The reason is the influence of the band section corners, negligible at short time, but contributing by an amount of the same order than the one from the boundary layers when their thickness $\delta$ becomes comparable to the width $b$. The total friction per unit length writes in fact (in the limit $h \ll b$ )

$$
\tau_{f}=2 \eta \frac{U_{0} b}{\sqrt{\pi \nu t}}\left(1+\frac{2 \sqrt{\nu t}}{\sqrt{\pi} b}\right)
$$

and is indeed twice that obtained by simply adding the plane boundary layers contributions when $\delta \simeq \sqrt{\nu t} \approx b$, a condition soon reached in the present case (see figure 7).

\section{(c) Dynamic buckling with a linear stress profile}

Because of skin friction, in glycerol, when the front reaches the clamped end its shape is approximately a straight line. Indeed, $\epsilon=\operatorname{erf}(x / 2 \sqrt{D T}) \sim x / 2 \sqrt{D T}$ for $x \ll \sqrt{D T}$. Thus as an approximate model, we consider the rebound of a linear front moving at a velocity $c$ towards the clamped end. When the stress free front reaches the clamped end at $t=0$, the strain profile has the form $\epsilon(x)=\epsilon_{0}(1-x / \ell)$ as shown in figure $9(\mathrm{i})$. 


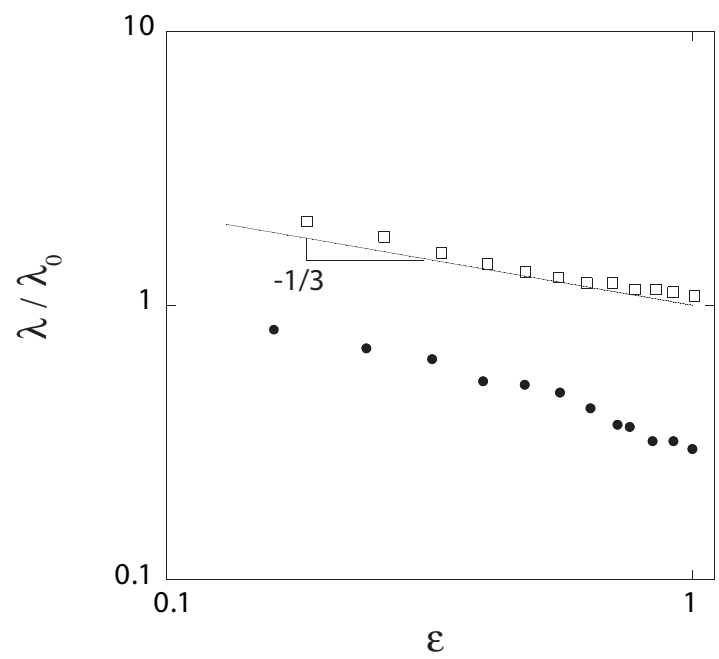

Figure 10. Evolution of the buckled wavelength with the initial stretching in log-log scale with $\lambda_{0}=2\left(\pi^{2} h^{2} \ell / 12\right)^{1 / 3} . \square$, measurements in glycerol $\eta=0.650 \mathrm{~Pa}$ s; $\bullet$, measurements in air. The continuous line shows the theoretical curve (equation 4.13) with no adjusted parameter.

At time $t$, the fronts rebounds and in the section behind the rebounding front $(0 \leq x \leq \ell-c t)$ the stretching is $\epsilon(x)=-2 \epsilon_{0} x / \ell$. At time $t$, the mean compressive force behind the front is $T_{m}=-($ Ebhct $/ \ell) \epsilon_{0}$. We consider that buckling starts when the front has reached the length $\lambda / 2$ where $\lambda=\left(\left|T_{m}\right| / 2 E I\right)^{1 / 2}$ is the wavelength selected by dynamic buckling and thus we obtain

$$
\lambda=2\left\{\frac{\pi^{2} h^{2} \ell}{12 \epsilon_{0}}\right\}^{1 / 3}
$$

The same method applied to the case of a discontinuous front results in the following expression of the buckled wavelength : $\lambda=\pi h \sqrt{2 / 3 \epsilon_{0}}$ as it is known and discussed in section $3(\mathrm{~b})$, considering $\epsilon_{0} \ll 1$. A satisfactory comparison with the experimental measurements of the buckled wavelength is shown in figure 10. This approach shows that taking into account the spreading of the front is sufficient to explain the greater observed wavelengths. Therefore, the increase of the buckled wavelength is not due to viscous effects involved during the dynamic buckling itself.

\section{Conclusion}

The main phenomena involved in the recoil of an initially stretched rubber band are the propagation of an axial-stress front, its rebound and the development of a buckling instability. A simple description of longitudinal and transverse elastic waves provides a good insight on the dynamics. The main point is that at early stage of the recoil, the wavelength is correctly predicted in this framework at least for moderate initial elongation (i.e. $\epsilon_{0}<1$ ).

At higher initial strain, the wavelength becomes of the order of the band thickness and the long wave description is no longer appropriate. Three dimensional 
deformations lead to even smaller wavelength. Once the band is wrinkled, the axial stress relaxes by a simple geometrical effect leading to a coarsening of the initial undulations.

When the rubber band is immersed in a fluid, the major effect is the spreading of the initial front due to boundary layer friction. The smoother stress profile leads to longer wavelength and a simple model based on a linear compressive strain profile gives a good estimate of the most amplified wavelength. Added mass effects slow down the instability but do not modify mode selection appreciably. The impact of both fluid viscosity and density on the instability development are quantified with appropriate dimensionless numbers.

It was not necessary to account for a possible nonlinear elastic response of the material.

\section{Appendix A. Dispersion relation for the buckling of a rod interacting with a surrounding fluid}

We derive the dispersion relation (equation 4.1) for the waves propagating along the rubber band in a viscous fluid, in two dimensions. In the reference (undeformed) state, the elastic rod is of infinite extent in the $x$ direction and its thickness in the $y$ direction is $h$. There are two fluids domain, denoted by the index 1 for the upper part ( $y>0$ in the reference configuration) and 2 for the lower part separated by the rubber band. The model is based on the linearized Navier Stokes equation for the two fluid domains.

$$
\begin{aligned}
\rho \frac{\partial U_{1,2}}{\partial t} & =-\frac{\partial P_{1,2}}{\partial x}+\eta\left(\frac{\partial^{2} U_{1,2}}{\partial x^{2}}+\frac{\partial^{2} U_{1,2}}{\partial y^{2}}\right) \\
\rho \frac{\partial V_{1,2}}{\partial t} & =-\frac{\partial P_{1,2}}{\partial y}+\eta\left(\frac{\partial^{2} V_{1,2}}{\partial x^{2}}+\frac{\partial^{2} V_{1,2}}{\partial y^{2}}\right)
\end{aligned}
$$

The fluid is incompressible and thus, for the pressure, we have $\nabla^{2} P_{1,2}=0$ and we look for $P_{1}$ and $P_{2}$ of the form

$$
\begin{aligned}
& P_{1}=p_{1}^{(0)}+p_{1} \exp (-k y) \exp (\sigma t-i k x) \\
& P_{2}=p_{2}^{(0)}+p_{1} \exp (k y) \exp (\sigma t-i k x)
\end{aligned}
$$

where we have used the condition that $P$ must remain finite at infinity. We look for $V_{1,2}$ of the form :

$$
V_{1,2}=v_{1,2}(y) \exp (\sigma t-i k x)
$$

Using these forms for $P_{1,2}$ and $V_{1,2}$ in equations (A 1) and (A 2) we have

$$
\begin{aligned}
q^{2} v_{1}-\frac{\partial^{2} v_{1}}{\partial y^{2}} & =-\frac{k}{\eta} p_{1} \exp (-k y) \\
q^{2} v_{2}-\frac{\partial^{2} v_{2}}{\partial y^{2}} & =\frac{k}{\eta} p_{2} \exp (-k y)
\end{aligned}
$$

where

$$
q^{2}=\frac{\rho \sigma}{\eta}+k^{2}
$$


Thus for $V_{1}$ and $V_{2}$, we have (using the condition that $V$ must remain finite)

$$
\begin{aligned}
& V_{1}=\left\{A_{1} \exp (-q y)+B_{1} \exp (-k y)\right\} \exp (\sigma t-i k x) \\
& V_{2}=\left\{A_{2} \exp (q y)+B_{2} \exp (k y)\right\} \exp (\sigma t-i k x)
\end{aligned}
$$

with

$$
p_{1}=\frac{\eta}{k}\left(q^{2}-k^{2}\right) B_{1}, \text { and } p_{2}=\frac{\eta}{k}\left(q^{2}-k^{2}\right) B_{2}
$$

We use the continuity equations

$$
\frac{\partial U_{1,2}}{\partial x}+\frac{\partial V_{1,2}}{\partial y}=0
$$

to obtain $U_{1,2}$

$$
U_{1,2}=-\frac{1}{i k} \frac{\partial V_{1,2}}{\partial y}
$$

We need to specify the boundary conditions at the interface between the fluid and rod. There are four of them:

- Assuming that the cross sections of the rod are moving along the $y$ direction without being stretched or compressed, the transverse displacement is therefore homogenous along a section. Then we deduce the kinetic conditions at both interfaces $(y= \pm h / 2)$ in the transverse direction

$$
\left.V_{1}\right|_{y=h / 2}=\left.V_{2}\right|_{y=-h / 2}=\frac{\partial \xi}{\partial t}
$$

- With a fluid initially at rest and in the slender slope limit, the difference of horizontal velocity across a section is

$$
\left.U_{1}\right|_{y=h / 2}-\left.U_{2}\right|_{y=-h / 2}=-h \frac{\partial^{2} \xi}{\partial t \partial x}
$$

- Then, neglecting the thickness of the rod $h$ versus the wavelength $\lambda=2 \pi / k$, the equation (A 15) leads to the kinetic condition in the axial direction

$$
\left.U_{1}\right|_{y=h / 2}=\left.U_{2}\right|_{y=-h / 2}
$$

- Moreover, we are looking for modes that are anti-symmetrical across the medium line, i.e. such that

$$
\Gamma_{x y, 1}=\Gamma_{x y, 2}
$$

where $\Gamma_{x y, 1,2}$ is the $x y$ term of the viscous stress tensor in the fluid. This condition also states that the shear stress at the rod surface is anti-symmetrical.

For the transverse motion of the rod, we use an Euler-Bernoulli model

$$
\left.\rho_{0} S \frac{\partial^{2} \xi}{\partial t^{2}}+E I \frac{\partial^{4} \xi}{\partial x^{4}}+T \frac{\partial^{2} \xi}{\partial x^{2}}+b \Delta\left[-P_{1,2}+\underline{\underline{n_{2}}} \cdot \underline{\underline{\left(\Gamma_{1,2}\right.}} \underline{\underline{n_{1,2}}}\right)\right]=0
$$


where the last term of the left hand side represents the fluid-stress difference between both sides of the rod. $\underline{\underline{\Gamma}}$ is the viscous stress tensor in the fluid and $n_{1,2}$ is the vector normal to the interface. $\Gamma$ in the fluid takes the form

$$
\stackrel{\underline{\Gamma_{1,2}}}{=}\left(\begin{array}{cc}
\eta \frac{\partial U_{1,2}}{\partial x} & \frac{\eta}{2}\left(\frac{\partial U_{1,2}}{\partial y}+\frac{\partial V_{1,2}}{\partial x}\right) \\
\frac{\eta}{2}\left(\frac{\partial U_{1,2}}{\partial y}+\frac{\partial V_{1,2}}{\partial x}\right) & \eta \frac{\partial V_{1,2}}{\partial y}
\end{array}\right)
$$

At leading order, we have $\underline{n_{1}}=-\underline{n_{2}}=(-\partial \xi / \partial x, 1)$ and thus,

$$
\left.\Delta\left[\underline{n_{2}} \cdot \underline{\underline{\Gamma_{1,2}}} \underline{n_{1,2}}\right)\right]=2 \eta\left(\left.\frac{\partial V_{2}}{\partial y}\right|_{y=h / 2}-\left.\frac{\partial V_{1}}{\partial y}\right|_{y=-h / 2}\right)
$$

Using these four conditions in equations A 6, A 7 and (A 14) we obtain a dispersion equation for the dynamic buckling of the rod in the fluid

$$
A_{1}-A_{2}=\left(B_{2}-B_{1}\right) \exp \left[\frac{(q-k) h}{2}\right]
$$

Using the form of $U_{1,2}$ (equation A 13) and (A 16) we obtain

$$
A_{1}+A_{2}=-\frac{k}{q}\left(B_{2}+B_{1}\right) \exp \left[\frac{(q-k) h}{2}\right]
$$

And finally from the relation between tangential stress (equation A 17) and using the form of $V$ (equations A 9 and A 10) and the form of $U$ (equation A 13), we get

$$
A_{1}-A_{2}=\frac{2 k^{2}}{q^{2}+k^{2}}\left(B_{2}-B_{1}\right) \exp \left[\frac{(q-k) h}{2}\right]
$$

Combining equations (A 21) and (A 23) and using equation (A 11), we have

$$
\begin{aligned}
A_{1} & =A_{2} \\
B_{1} & =B_{2} \\
p_{1} & =-p_{2}
\end{aligned}
$$

Using equation (A 22) we find :

$$
A_{1}=-\frac{k}{q} B_{1} \exp \left[\frac{(q-k) h}{2}\right]
$$

We write $\Delta\left(-P_{1,2}+\underline{n_{2}} \cdot\left(\underline{\Gamma_{1,2}} \underline{n_{1,2}}\right)\right)$ in terms of $B_{1}$ :

$$
\begin{aligned}
\Delta & \left.\left(-P_{1,2}+\underline{n_{2}} \cdot \underline{\underline{\underline{\Gamma_{1,2}}}} \frac{n_{1,2}}{}\right)\right) \\
& =\left\{\left(p_{1}-p_{2}\right) e^{-\frac{k h}{2}}+\eta q\left(A_{1}+A_{2}\right) e^{-\frac{q h}{2}}+\eta k\left(B_{1}+B_{2}\right) e^{-\frac{k h}{2}}\right\} e^{\sigma t-i k x} \\
& =\left\{2 \frac{\eta\left(q^{2}-k^{2}\right)}{k} B_{1} e^{-\frac{k h}{2}}\right\} e^{\sigma t-i k x}
\end{aligned}
$$


The expression of $\xi$ is obtained from the transversal boundary condition (equation A 14)

$$
\xi=\frac{1}{\sigma}\left\{A_{1} e^{-\frac{q h}{2}}+B_{1} e^{-\frac{k h}{2}}\right\} e^{\sigma t-i k x}
$$

Introducing these expression in (A 18) and using the relation (A 21) we obtain the dispersion equation

$$
\left(\rho_{0} S+2 \frac{\rho b}{k}\right) \sigma^{2}+2 b \eta(k+q) \sigma+E I k^{4}-T k^{2}=0
$$

In dimensionless form this relation reads

$$
\left\{k_{*}+4 M\right\} \sigma_{*}^{2}+4 \chi k_{*}\left\{k_{*}+\left(\frac{M}{\chi} \sigma_{*}+k_{*}^{2}\right)^{1 / 2}\right\} \sigma_{*}+4 k_{*}^{3}\left(k_{*}^{2}-1\right)=0
$$

with

$$
k_{*}=\frac{k}{k_{c}}, \quad \sigma_{*}=\frac{\sigma}{\sigma_{m}}
$$

The two dimensionless coefficients are

$$
\chi=\left(\frac{\eta^{2} b}{\rho_{0} T h}\right)^{1 / 2} \text { and } \quad M=\frac{\rho \lambda_{c}}{4 \pi \rho_{0} h}
$$

\section{References}

Bouasse, H. and Carrière, Z. 1903. Sur les courbes de traction du caoutchouc vulcanisé. Annales de la faculté des sciences de Toulouse, 2e série, 5, 257 - 283.

Euler, L. 1744. Addidentum I de curvis elasticis, methodus inveniendi lineas curvas maximi minimivi proprietate gaudentes. In Opera Omnia I, 231-297. Lausanne, 1744.

Gladden, J. R., Handzy, N.Z., Belmonte, A. and Villermaux, E. 2005. Dynamic buckling and fragmentation in brittle rods. Phys. Rev. Lett., 94, 035503.

Graff, K. G. 1975. Wave motion in elastic solids. New York: Dover Publications.

Kolsky, H. 1949 . An investigation of the mechanical properties of materials at very high rates of loading. Proc. Phys. Soc. B, 62, 676-700.

Lamb, H. 1932. Hydrodynamics. Cambridge University Press.

Lindberg, H. E. 1965. Impact buckling of a bar. J. Appl. Mech., 32, 315-322.

Love, A. E. H. 1944. A treatise on the mathematical theory of elasticity. 4th edn. New York: Dover Publications.

Mullins, L. 1947. Effect of stretching on the properties of rubber. J. Rubber Res. 16, 275-289.

Saint-Venant, M. and Flamant, A. 1883. Résistance vive ou dynamique des solides. Représenttaion graphique des lois du choc longitudinal, subi à une des extrémités par une tige ou barre prismatique assujettie à l'extrémité opposée. C. R. Acad. Sci., 97, 127-133.

Stokes, G. G. 1850 On the effect of the internal friction of fluids on the motion of pendulums. Trans. Camb. Phil. Soc., IX, 8, sec 52. 